\title{
Colliding gastric and intestinal phenotype well-differentiated adenocarcinoma of the stomach developing in an area of MALT-type lymphoma
}

\author{
Mitsukuni Suenaga ${ }^{1}$, Kei-ichiro Ohta ${ }^{1}$, Masataka Toguchi ${ }^{1}$, Takahiro Sato ${ }^{1}$, Shigekazu Ohyama ${ }^{1}$, \\ Toshiharu Yamaguchi ${ }^{1}$, Tetsuichiro Muto ${ }^{1}$, Akio Yanagisawa ${ }^{2}$, and Yo Kato ${ }^{2}$ \\ ${ }^{1}$ Division of Gastroenterological Surgery, Cancer Institute Hospital, Tokyo, Japan \\ ${ }^{2}$ Department of Pathology, Cancer Institute, 1-37-1 Kamiikebukuro, Toshima-ku, Tokyo 170-8455, Japan
}

\begin{abstract}
A 73-year-old man presented with an abnormal gastric shadow during a check-up of atomic bomb survivors. Radiological examination and endoscopy of the upper gastrointestinal tract revealed a protruding tumor, type 0 -I+IIa, on the lesser curvature of the midstomach. An initial diagnosis of early gastric cancer was made and a segmental gastrectomy was planned. However, distal gastrectomy with D3 lymph node dissection was necessary, because intraoperative frozen section showed that the paraaortic lymph nodes (N3) were positive for cancer. The tumor in the resected specimen was, microscopically, a well-differentiated tubular adenocarcinoma (tub1) with pT2 (MP), pN3, ly2, and v1, in final (f) stage IV. The tumor cells of the type 0 -I segment appeared as gastric phenotype and those of the type 0-IIa segment as intestinal phenotype. The border between the two was distinct. The tumor had focally invaded the muscularis propria where only the gastric phenotype was shown and the histological type became less differentiated. Thus, special attention should be paid to possible unexpected deep-wall invasion and lymph node metastasis in well-differentiated adenocarcinomas of the gastric phenotype. Further, in this patient, diffusely proliferating low-grade lymphoma was also observed incidentally in the gastric mucosa within and around the carcinoma. This was diagnosed as mucosa-associated lymphoid tissue (MALT)type lymphoma with aberrant expression of BCL10. Finally, this case was considered to be a colliding gastric and intestinal phenotype well-differentiated adenocarcinoma of the stomach developed in an area involved by MALT-type lymphoma. Because no Helicobacter pylori was detected throughout the mucosae and the patient had no history of its infection, the three tumors may have developed under the same conditions as those seen in Helicobacter pylori infection, but without this infection.
\end{abstract}

Key words Gastric carcinoma - Gastric phenotype adenocarcinoma $\cdot$ MALT-type lymphoma

Offprint requests to: $\mathrm{Y}$. Kato

Received: March 12, 2003 / Accepted: August 25, 2003

\section{Introduction}

Histologically, gastric cancer is generally classified into two types, i.e., well-differentiated type, originating from intestinal metaplasia of the stomach or allied mucosa, and poorly differentiated type, originating from the gastric mucosa proper. Well-differentiated type carcinoma, consisting of cells with a morphologic resemblance to foveolar epithelia or pyloric gland cells, has recently attracted interest, and has been classified as a new category, i.e., gastric phenotype carcinoma, different from the usual intestinal phenotype carcinoma. We present a rare case of colliding gastric and intestinal phenotype well-differentiated adenocarcinoma of the stomach, that was revealed by histological examination to have developed incidentally in an area of mucosa-associated lymphoid tissue (MALT)-type lymphoma. The pathological description we use follows that in the Japanese classification of gastric carcinoma, 13th edition [1].

\section{Case report}

A 73-year-old man presented with an abnormal gastric shadow during a routine check-up for atomic bomb survivors. This was initially diagnosed as gastric cancer by upper gastrointestinal endoscopy. The patient was referred to our hospital for detailed examination and treatment; he had no gastrointestinal symptoms and no history of Helicobacter pylori eradication therapy. No remarkable abnormalities were detected on physical examination or laboratory analysis, which included tumor markers such as carcinoembryonic antigen and carbohydrate antigens 19-9 and 125. Radiology and endoscopy of the upper gastrointestinal tract revealed a protruding tumor, type $0-\mathrm{I}+\mathrm{IIa}, 2.4 \mathrm{~cm}$ in diameter, on the lesser curvature of the midstomach (Fig. 1a). Histological examination of biopsy specimens revealed gastric phenotype well-differentiated tubular adeno- 
carcinoma (tub1) in the type 0 -I segment and intestinal phenotype in the type 0-IIa segment (Fig. 1b,c). Computed tomography scanning and ultrasonography of the abdomen did not detect any metastatic lesions of the gastric cancer throughout the body.

The patient was admitted to our department, and a segmental gastrectomy was planned, based on our initial diagnosis of early clinical (c) stage IA gastric cancer. However, distal gastrectomy with D3 lymph node dissection was necessary because paraaortic lymph nodes (N3) were positive for cancer by intraoperative frozen section diagnosis. However, the patient succumbed to hepatic failure resulting from lymph node metastases involving the hepatic hilum and to respiratory failure due to multiple lung metastases 23 months after the surgery.

\section{Macroscopic and pathological findings}

Macroscopic and microscopic examination revealed the gastric tumor to be a well-differentiated tubular adenocarcinoma (tub1) of type 0 -I + IIa, $2.0 \times 2.3 \mathrm{~cm}$, showing depth of invasion to the muscularis propria (pT2, MP), moderate lymphatic invasion (ly2), minimal venous invasion (v1), INF $\alpha$, with metastasis to group 3 lymph nodes (pN3), and thus, final stage (f stage) IV (Fig. 2a,b). With hematoxylin and eosin staining (H\&E) and Alcian blue-periodic acid Schiff staining (AB-PAS), the type 0-I segment was composed of cuboidal or columnar cells characterized by clear mucous cytoplasm similar to foveolar epithelium, classified as a gastric phenotype tumor (area G in Fig. 3a). The type 0-IIa segment was composed of eosinophilic columnar cells that formed a well-differentiated glandular structure, together with goblet cells, being indicative of intestinal phenotype adenocarcinoma (area I in Fig. 3a). The border between the two tumor segments was distinct (area B in Fig. 3a, Fig. 3b1). Furthermore, focal invasion of the muscularis propria was apparent in the area of the gastric phenotype tumor and it was accompanied by a histological shift to a less differentiated type, but the gland formation was fairly well preserved even in the advancing area of the tumor (area F in Fig. 3a; Fig. 3c1). In the mucosa surrounding the carcinoma, diffusely proliferating low-grade lymphoma cells were observed incidentally with lymphoepithelial lesions. The lymphoma cells, slightly larger than mature lymphocytes, with irregularly shaped nuclei, corresponded to centrocytelike cells (CCL) of MALT-type lymphoma (area M in Fig. 3a; Fig. 4a,b). There was no evidence of Helicobacter pylori infection throughout the mucosae, and rapid urease test was negative. In addition, immunohistochemical staining with rabbit anti-Helicobacter pylori (Dako, Glostrup, Denmark) also failed to detect Helicobacter pylori infection.
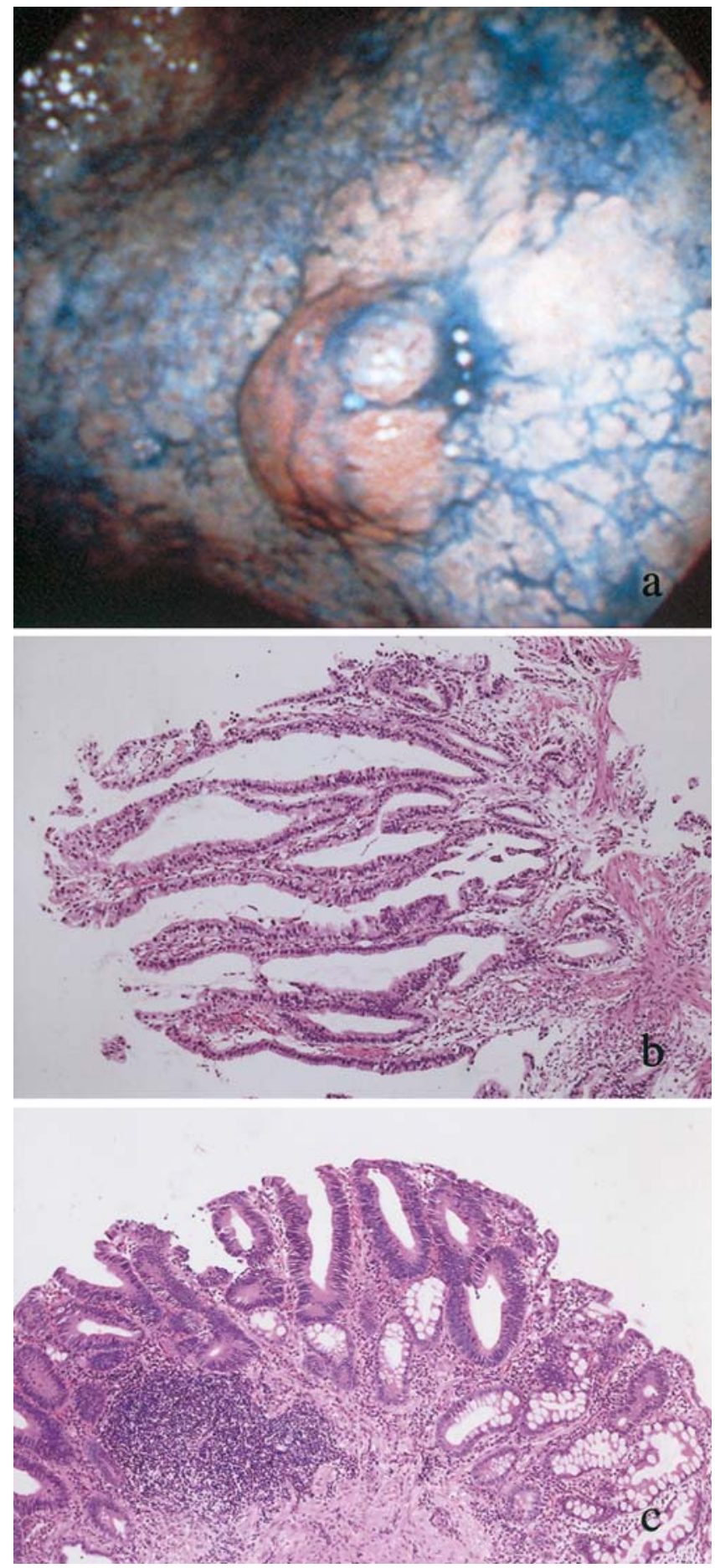

Fig. 1a-c. Endoscopic and biopsy findings. a Chromoendoscopy with indigocarmine, showing a protruding tumor, type 0-I+IIa, $2.4 \mathrm{~cm}$ in diameter, on the lesser curvature of the midstomach. b Biopsy section from type 0-I segment, showing gastric phenotype well-differentiated tubular adenocarcinoma (tub1). c Biopsy section from type 0-IIa segment, showing intestinal phenotype well-differentiated tubular adenocarcinoma 

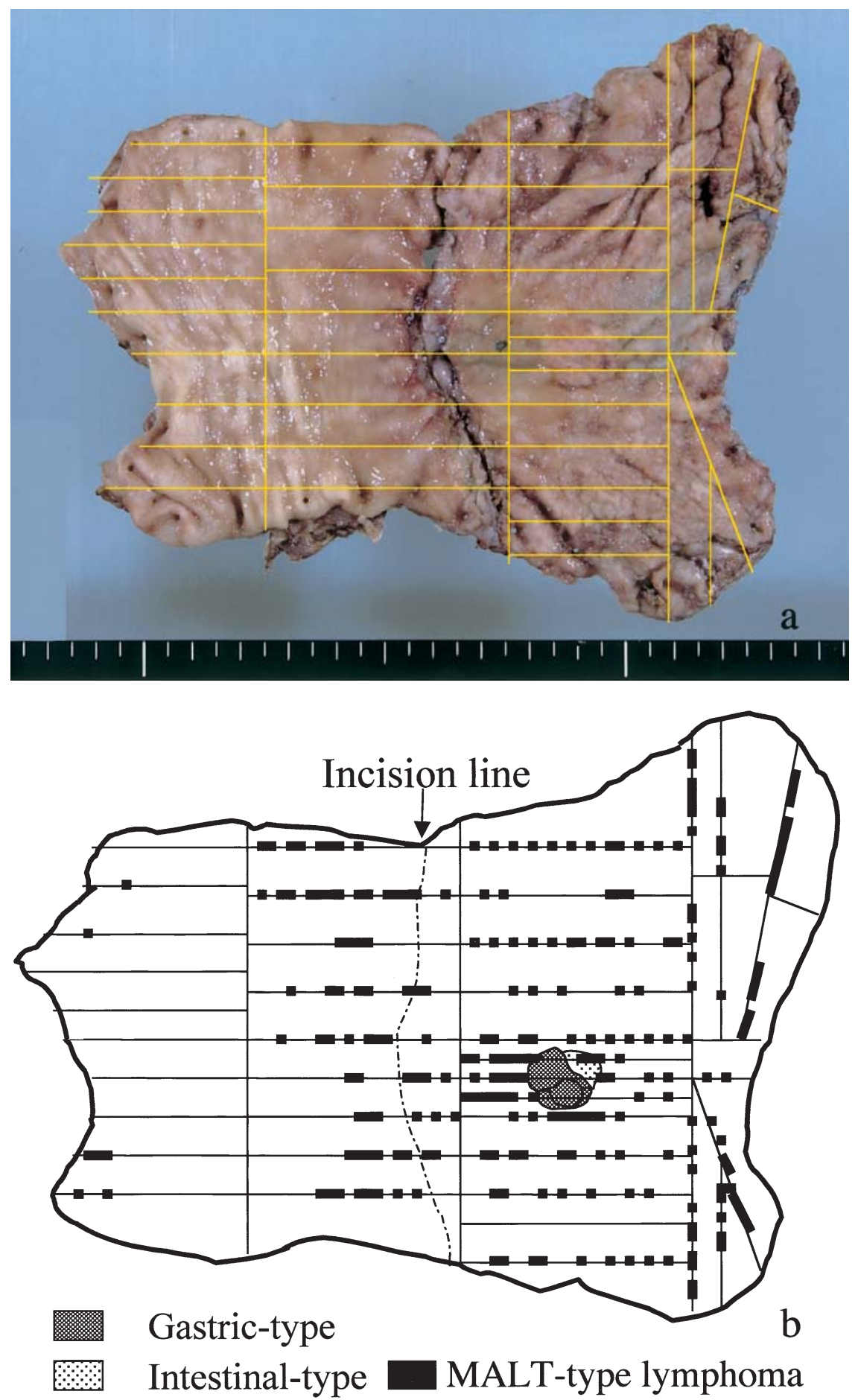

Fig. 2. a Resected specimen with section lines, showing a type 0 -I+IIa tumor on the lesser curvature of the mid-stomach. b Schematic presentation of the spread of three tumors. MALT, mucosa-associatedlymphoid tissue

\section{Immunohistochemical findings}

Several histochemical and immunohistochemical stainings (Fig. 3) were employed to differentiate the two phenotypes in the carcinoma: human gastric mucin (mouse monoclonal antibody, clone 45M1; Novocastra Laboratories, Newcastle upon Tyne, UK), Con A (concanavalin A from Canavalia ensiformis; Sigma Chemical St. Louis, MO, USA), Muc2 glycoprotein (mouse monoclonal antibody, clone Ccp58; Novocastra Laboratories), and CD10 (mouse monoclonal antibody, clone 56C6; Novocastra Laboratories). The antibody against $45 \mathrm{M} 1$ reacted in areas $\mathrm{G}$ and $\mathrm{F}$, but not in area I as shown in Fig. 3b2, c2. ConA-positive cells were scat- 

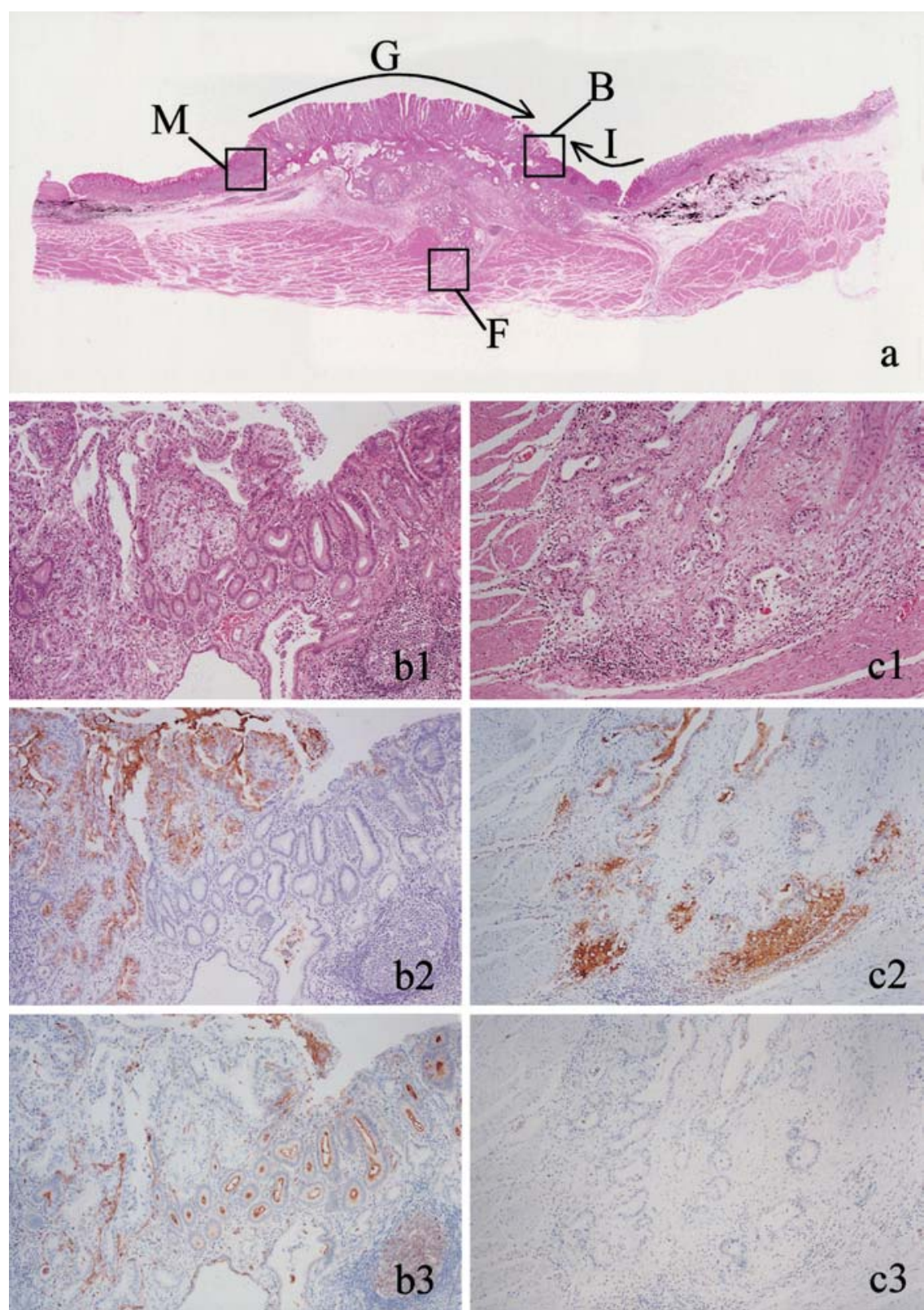

Fig. 3a-c3. Histological and immunohistochemical findings of three tumors. a Low-power view of the tumor $(\mathrm{H} \& \mathrm{E})$, showing the gastric $(G)$ and intestinal $(I)$ phenotype well-differentiated adenocarcinomas colliding. $B$ Indicates the border of areas $G$ and $I ; F$, focal invasion (MP); $M$, MALT-type lymphoma. b1 Highpower view of $\mathbf{B}$ in a $(\mathrm{H} \& \mathrm{E})$; b2 $45 \mathrm{M} 1$; b3 CD10. c1 High-power view of $F$ in a (H\&E); c2 45M1; c3 CD10. 45M1 was bound in areas $G$ and $F$. The epithelial brush borders were stained with CD10 in area I. b1-3; c1-3 $\times 25$ tered only in area G, but not in area I. The epithelial brush borders were stained only with CD10 in area I, but not in areas G and F (Fig. 3b3, c3). However, Muc2 was not expressed in any areas examined. Concerning the lymphoma, expression of the following markers was examined: UCHL-1 (monoclonal mouse anti-human T cell, CD45R0, clone UCHL-1; Dako), L26 (monoclonal mouse anti-human B cell, CD20, clone L26; Dako), CD5 (mouse monoclonal antibody, clone 4C7; Novocastra Laboratories), cyclin D1 (mouse monoclonal IgG1 antibody; Santa Cruz Biotechnology, Santa Cruz, CA, USA), BCL2 (monoclonal mouse anti-human BCL2 oncoprotein, clone 124; Dako), and BCL10 (monoclonal mouse anti-BCL10 antibody; Zymed Laboratories,
South San Francisco, CA, USA). All markers but L26, BCL2, and BCL10 were negative (Fig. 4c-h), suggesting MALT-type lymphoma with aberrant expression of BCL10. From the data, we interpreted that this case was a colliding gastric and intestinal phenotype welldifferentiated adenocarcinoma developing within an area diffusely involved by MALT-type lymphoma.

\section{Discussion}

Gastric cancer is generally divided into two major histological types, i.e., well-differentiated type, originating from intestinal metaplasia of the stomach or the allied 

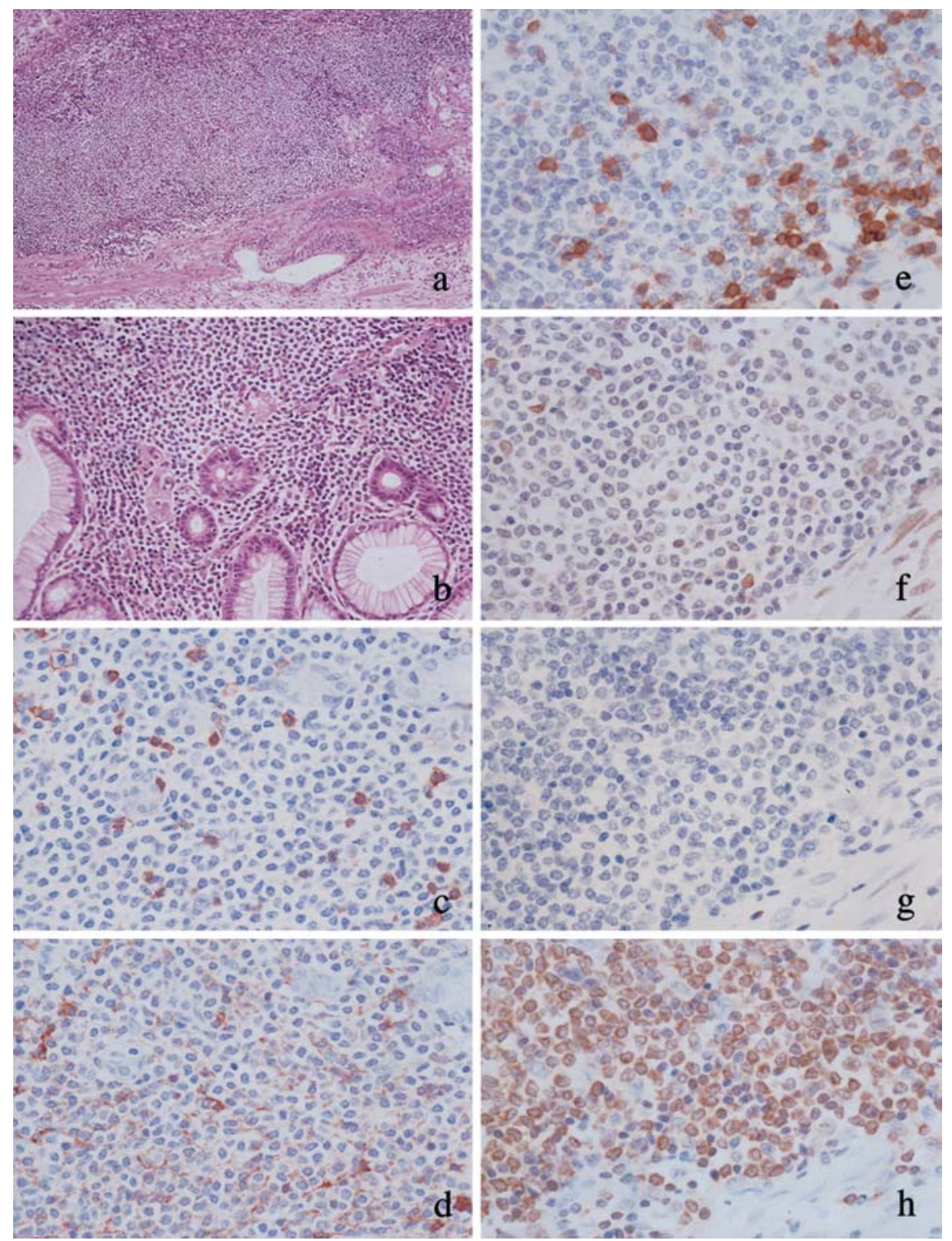

Fig. 4a-h. Histological and immunohistochemical findings of MALT-type lymphoma ( $M$ in Fig. 3a). a Diffusely proliferating low-grade lymphoma cells involving the gastric mucosa $(\mathrm{H} \& \mathrm{E})$; b lymphoepithelial lesions (H\&E); c UCHL-1; d L26; e CD5; f cyclin D1; g CD10; h BCL2. All but markers L26 and BCL2 were negative. $\mathbf{a} \times 25$; b $\times 75$; c-h $\times 150$ mucosa, and poorly differentiated type, originating from the gastric mucosa proper [2]. The former corresponds to the intestinal-type, whereas the latter corresponds to the diffuse-type according to Lauren's classification [3]. However, differentiated carcinomas, consisting of cells that are morphologically similar to foveolar epithelium or pyloric gland cells, have been recently classified as a new category, i.e., gastric phenotype different from the usual intestinal phenotype [4-13]. The phenotype characterized by mucin expression in gastric adenocarcinoma has been immunohistochemically classified into four types: gastric, intestinal, combined gastric and intestinal phenotype, and the absence of mucin [11,12]. Further, carcinoma of the gastric phenotype has a tendency to more readily show submucosal invasion, lymphatic or venous invasion, and lymph node metastasis than that of the intestinal phenotype
[13]. In the present patient, the diagnosis was welldifferentiated tubular adenocarcinoma occurring between the fundus and pyloric gland mucosa, and consisting of both gastric and intestinal phenotypes, which showed a distinct border between them in an apparently single tumor. This tumor was considered to be a colliding carcinoma with two different origins rather than a single-cell-origin carcinoma. To clarify this, genetic analysis would be necessary. It was quite noticeable in this patient that only the gastric phenotype, not the intestinal phenotype, had invaded the deeper layer of the gastric wall and caused lymph node metastases. Although this finding is, as a whole, consistent with previous reports, it was rather peculiar that, in this patient, the glandular structure was maintained even in the advancing area of the tumor without changing into poorly differentiated type. Nevertheless, one should be 
aware that there may be unexpected deep-wall invasion and lymph node metastasis in well-differentiated adenocarcinomas of the gastric phenotype.

MALT-type lymphoma is a low-grade B-cell lymphoma derived from MALT and is rarely associated with gastric carcinoma [14-16]. In patients with coexistent lymphoma and carcinoma, the two neoplasms are usually located separately, lymphoma is advanced, and carcinoma is of the well-differentiated type [17-20]. Interestingly, in our patient, the carcinoma had developed completely within an area of lymphoma. Helicobacter pylori infection has been detected more frequently in patients with two different coexisting tumors (i.e., lymphoma and carcinoma) than in patients with either of the tumors particularly in those with carcinoma. This suggests that Helicobacter pylori infection may be a causal factor in the development of these two neoplasms. In the present patient, however, this would not have been a causal factor, because no Helicobacter pylori was detected. The relationship between Helicobacter pylori infection and intestinal metaplasia is well known: Helicobacter pylori causes atrophic gastritis, including intestinal metaplasia, but disappears from the gastric mucosa with an increase of intestinal metaplasia. Thus, it is likely that the incidence of Helicobacter pylori infection is lower in a stomach with well-differentiated adenocarcinoma than in one with poorly differentiated adenocarcinoma [21-23]. In our patient, the degree of intestinal metaplasia in the resected specimen was moderate, but Helicobacter pylori was not detected in any part of the gastric mucosa, although the nonmetaplastic or gastric proper mucosa was fairly well maintained. So it is possible that, in this patient no Helicobacter pylori infection had existed from youth, and factors other than Helicobacter pylori had caused the atrophic gastritis.

At the age of 18 years, the patient had been within $1.5 \mathrm{~km}$ of ground zero during an atomic blast. It is controversial whether the incidence of gastric carcinoma is higher in persons exposed to an atomic blast in comparison to nonexposed subjects [24-27], but there is a report that its incidence is highest in persons exposed within a $2.0-\mathrm{km}$ radius of ground zero [25]. For this reason, the gastric carcinogenesis, as well as the development of atrophic gastritis in our patient could be related to exposure to the atomic blast. On the other hand, no relationship between MALT-type lymphoma and atomic bomb exposure has been indicated. But, recently, $\mathrm{t}(11 ; 18)(\mathrm{q} 21 ; \mathrm{q} 21)$ and $\mathrm{t}(1 ; 14)(\mathrm{p} 22 ; \mathrm{q} 32)$ translocations have been reported to be associated with MALT-type lymphoma. The former translocation results in the fusion protein AP I2-MALT1, and the latter results in the fusion protein of BCL10 and IgH. These fusion transcripts are thought to be molecular markers for MALTtype lymphoma not responding to Helicobacter pylori eradication and highly correlated with aberrant nuclear BCL10 expression, which may serve as a screening tool for the fusion [28-30]. In our patient, aberrant BCL10 was expressed in the nuclei of the MALT-type lymphoma, suggesting the possibility of such fusion being present. Thus, it is likely that the MALT-type lymphoma in this patient had developed independently of Helicobacter pylori infection, or was related to exposure to the atomic bomb blast. So the three tumors, i.e., the gastric phenotype carcinoma, the intestinal phenotype adenoocarcinoma, and the MALT-type lymphoma, may have developed in the same conditions, as those seen with Helicobacter pylori infection, but without this infection.

Acknowledgments We appreciate very much that Professor T. Fujimori performed the BCL10 immunostaining of MALT-type lymphoma.

\section{References}

1. Japanese Gastric Cancer Association. Japanese classification of gastric carcinoma. 13th ed. Tokyo: Kanehara; 1999. pp. 4-6.

2. Nakamura K, Sugano H, Takagi K. Carcinoma of the stomach in incipient phase: its histogenesis and histological appearance. Gann 1968;59:251-8.

3. Lauren $P$. The two histological main types of gastric carcinoma: diffuse and so-called intestinal-type carcinoma. Acta Pathol Microbiol Scand 1965;64:31-49.

4. Egashira Y, Shimoda T, Ikegami M. Mucin histochemical analysis of minute gastric differentiated adenocarcinoma. Pathol Int 1999; 49:55-61.

5. Kushima R, Stolte M, Dirks K, Vieth M, Okabe H, Borchard F, et al. Gastric-type adenocarcinoma of the duodenal second portion histogenetically associated with hyperplasia and gastric-foveolar metaplasia of Brunner's glands. Virchows Arch 2002;440:655-9.

6. Hattori T, Kushima R. Gastric differentiated stomach adenocarcinoma; review (in German). Pathologe 2001;22:97-104.

7. Kabashima A, Yao T, Sugimachi K, Tsuneyoshi M. Gastric or intestinal phenotypic expression in the carcinomas and background mucosa of multiple early gastric carcinomas. Histopathology 2000;37:513-22.

8. Koseki K, Takizawa T, Koike M, Ito M, Nihei Z, Sugihara K. Distinction of differentiated type early gastric carcinoma with gastric type mucin expression. Cancer 2000;89:724-32.

9. Tatematsu M, Ichinose M, Miki K, Hasegawa R, Kato T, Ito N. Gastric and intestinal phenotypic expression of human stomach cancers as revealed by pepsinogen immunochemistry and mucin histochemistry. Acta Pathol Jpn 1990;40:494-504.

10. Kushima R, Hattori T. Histogenesis and characteristics of gastrictype adenocarcinoma in the stomach. J Cancer Res Clin Oncol 1993;120:103-11.

11. Shimoda T, Fujisaki J, Kashimura H, Ikegami M, Ishii T, Matsui $\mathrm{T}$, et al. Histological type of gastric carcinoma in relation to the mode of intramural spreading of cancer cells (in Japanese). Stomach and Intestine 1991;26:1125-34.

12. Kiyoko K, Yanagisawa A, Ninomiya $\mathrm{Y}$, Inoshita N, Kato $\mathrm{Y}$. Characteristics of differentiated-type carcinoma with gastric phenotype of the stomach (in Japanese). Stomach and Intestine 1999; 34:487-94.

13. Koseki K, Takizawa T, Koike M, Funata N, Hishima T, Hakuma $\mathrm{T}$, et al. Subclassification of well differentiated gastric cancer with 
reference to biological behavior and malignancy, gastric type vs intestinal type, and papillary carcinoma vs tubular carcinoma (in Japanese). Stomach and Intestine 1999;34:507-12.

14. Isaacson $\mathrm{P}$, Wright $\mathrm{DH}$. Malignant lymphoma of mucosaassociated lymphoid tissue: a distinctive type of B-cell lymphoma. Cancer 1983;52:1410-6.

15. Isaacson $\mathrm{P}$, Wright DH. Extranodal malignant lymphoma arising from mucosa-associated lymphoid tissue. Cancer 1984;53:251524.

16. Ito C, Kato M, Yamamoto T, Ota N, Okuhara T, Mabuchi K, et al. Study of stomach cancer in atomic bomb survivors. Report of histological findings and prognosis. J Radiat Res 1989;30:164-75.

17. Kanamoto K, Aoyagi K, Nakamura S, Hizawa K, Suekane H, Sakamoto K, et al. Simultaneous coexistence of early adenocarcinoma and low-grade MALT lymphoma of the stomach associated with Helicobacter pylori infection: a case report. Gastrointest Endosc 1998;47:73-5.

18. Mochizuki Y, Makino T, Yamazaki Y, Suda T, Takemura H, Nakamura N. Synchronous gastric adenocarcinoma and MALTtype lymphoma with Helicobacter pylori infection. A case report (in Japanese). Jpn J Gastroenterol Surg 2000;33:605-9.

19. Sakatoku M, Ietsugu K, Nakashima H, Kiyohara K, Kosugi M, Terahata S. A case of early gastric endocrine cell carcinoma, gastric lymphoma and early gastric cancer (in Japanese). Nippon Rinshogeka Gakkai Zasshi (J Jpn Surg Assoc) 2001;62:2913-17.

20. Takahashi H, Tomita T, Hayakawa H. Synchronous early gastric adenocarcinoma and MALT-lymphoma with Helicobacter pylori infection (in Japanese). A case report. Jpn J Gastroenterol Surg 2001;34:571-5.

21. Kato Y, Aizu K, Barreto RZ, Maruyama M. The relationship between $H$. pylori infection, intestinal metaplasia and gastric cancer development. In: Kimura K, Sakaki N, editors. Helicobacter pylori and gastritis and gastric cancer (in Japanese). Tokyo: Igaku-shoin; 1996. pp. 208-14.
22. Sipponen P. Gastric cancer: pathogenesis, risk, and prevention. J Gastroenterol 2002;37 (Suppl 13):39-44.

23. Sipponen P, Kimura K. Intestinal metaplasia, atrophic gastritis and stomach cancer: trends over time. Eur J Gastroenterol Hepatol 1994;6 (Suppl 1):79-83.

24. Iwasaki K, Kawamoto K, Shimokawa I, Matsuo T, Ikeda T. Epidemiological studies on gastric cancer in Nagasaki (in Japanese). Gan No Rinsho (Clin Med Cancer) 1984;30:1746-54.

25. Suehiro S, Nagasue N, Abe S, Ogawa Y, Sasaki Y. Carcinoma of the stomach in atomic bomb survivors. A comparison of clinicopathologic features to the general population. Cancer 1986;57: 1894-8.

26. Kai M, Leubeck EG, Moolgavkar SH. Analysis of the incidence of solid cancer among atomic bomb survivors using a two-stage model of carcinogenesis. Radiat Res 1997;148:348-58.

27. Little MP, Muirhead CR, Charles MW. Describing time and age variations in the risk of radiation-induced solid tumour incidence in the Japanese atomic bomb survivors using generalized relative and absolute risk models. Stat Med 1999;18:17-33.

28. Maea B, Demunter A, Peeters B, De Wolf-Peeters C. BCL10 mutation does not represent an important pathogenic mechanism in gastric MALT-type lymphoma, and the presence of the API2MALT fusion is associated with aberrant nuclear BCL10 expression. Blood 2002;99:1398-404.

29. Nakamura T, Nakamura S, Yokoi T, Ohashi K, Seto M. Clinicopathologic comparison between the API2-MALT1 chimeric transcript-positive and -negative gastric low-grade B-cell lymphoma of mucosa-associated lymphoid tissue type. Jpn J Cancer Res 2002;93:677-84.

30. Okabe M, Inagaki H, Oshima K, Yoshino T, Li C, Eimoto T, et al. API2-MALT1 fusion defines a distinctive clinicopathological subtype in pulmonary extranodal marginal zone B-cell lymphoma of mucosa-associated lymphoid tissue. Am J Pathol 2003;162:111322. 